\title{
Excitation of Large-Amplitude Localized Nonlinear Waves by the Interaction of Kinks of the Sine-Gordon Equation with Attracting Impurity
}

\author{
A. M. Gumerov, E. G. Ekomasov, R. V. Kudryavtsev, M. I. Fakhretdinov
}

The generation and evolution of localized waves on an impurity in the scattering of a kink of the sine-Gordon equation are studied. It is shown that the problem can be considered as excitation of oscillations of a harmonic oscillator by a short external impulse. The external impulse is modeled by the scattering of a kink on an impurity. The influence of the modes of motion of a kink on the excitation energy of localized waves is numerically and analytically studied. The method of collective coordinate for the analytical study is used. The value of this energy is determined by the ratio of the impurity parameters and the initial kink velocity. It is shown that the dependence of the energy (and amplitude) of the generated localized waves

Received January 27, 2019

Accepted March 21, 2019

This work was supported by Act 211 of the Government of the Russian Federation, contract No. 02.A03.21.0011. For A. M. Gumerov and R. V. Kudryavtsev the work was supported by the RFBR grant, project 18-31-00122.

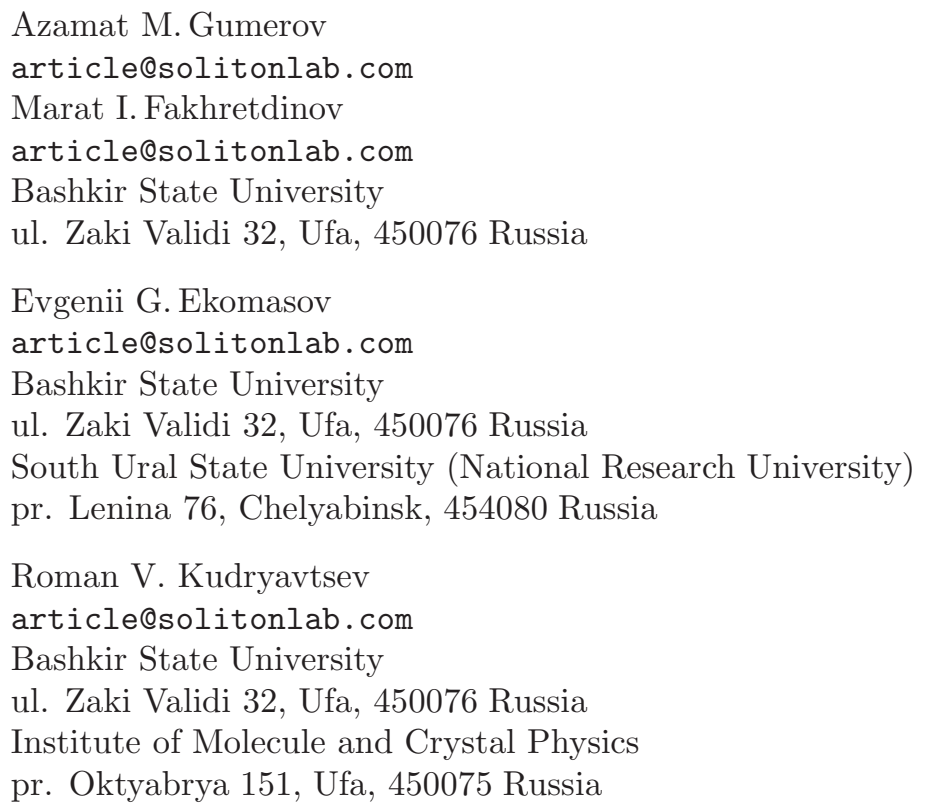


on the initial kink velocity has only one maximum. This behavior is observed for the cases of point and extended impurities. Analytical expression for the amplitude of the localized wave in the case of point impurity is obtained. This allows controlling the excitation energy of localized waves using the initial kink velocity and impurity parameters. The study of the evolution of localized impurities under the action of an external force and damping has shown a good agreement with the nondissipative case. It is shown that small values of the external force have no significant effect on the oscillations of localized waves. An analytical expression for the logarithmic decrement of damping is obtained. This study may help to control the parameters of the excited waves in real physical systems.

Keywords: sine-Gordon equation, impurity, kink, wave generation

\section{Introduction}

Soliton solutions of nonlinear differential equations attract increased attention of researchers because of an increasing use in physical applications [1-18]. For example, solitons of the sineGordon equation(the SGE) are frequently used. Only in solid state physics they describe domain boundaries in magnets, dislocations in crystals, fluxons in Josephson junctions. The sine-Gordon equation appears in the modeling of wave processes in rocks, molecular biology, models of field theory and particle physics $[2,3]$. Accounting for the influence of perturbations on the SGE solutions leads to a change in the structure of solitons and to the excitation of the internal degrees of freedom of solitons. It is important to study the SGE solutions considering the perturbations arising in real physical applications. The effect of small perturbations on the SGE solutions can be studied using a well-developed perturbation theory for solitons [5, 19, 20]. The effect of perturbations can generally be investigated only with the help of numerical methods [20-23]. For example, the influence of a coordinate- and time-dependent external force has been studied $[24-26]$.

Numerous works have studied spatially dependent perturbations in the sine-Gordon model [5, 6, 18-43]. Many papers study the effect of spatial modulation of the periodic potential (or the presence of impurities in the system) on the dynamics of the SGE solitons $[1,2,5,6,19,28]$. The SGE model with impurities describes, for example, a multilayer ferromagnet [40-43]. The importance of impurity modes in the kink dynamics was shown in $[5,28,36]$. The structure and properties of localized nonlinear waves excited on an impurity were analyzed numerically in $[21,23,32]$. The case of several point impurities that are of interest in some physical applications was considered in [44] and even the case of spatially modulated harmonic potential was considered [31]. The possibility of exciting localized waves as a result of the kinkimpurity interaction has been also investigated in some cases [5,6]. At first, a simple classical particle model was used for the theoretical description of the kink-impurity interaction when the excitation of an impurity mode was neglected, i.e., a localized oscillatory state on the impurity [5]. Accounting for the excitation of impurity modes in the kink-impurity interaction leads to an interesting effect, i.e., the reflection of a kink by an attracting impurity due to the resonance energy exchange between the translational mode of the kink and the impurity mode. The work [22] has shown that this effect can be observed under certain conditions even in the presence of damping that always occurs in real physical systems. The value of energy consumed for the excitation of localized waves by the kink will determine the value of a new contribution to the effective damping of a moving kink [45]. It may change depending on its initial velocity. It is known that, in this case, the frequency of excited localized nonlinear waves is practically 
independent of the initial kink velocity. But this velocity must influence the amplitude of the excited wave. In this paper, we study the problem of the possibility of controlling the structure, dynamics, and energy of localized nonlinear waves excited by a moving kink on an impurity.

\section{Basic equations and results}

Consider a system determined by the Lagrangian

$$
L=\int_{-\infty}^{+\infty}\left\{\frac{1}{2}\left(\frac{\partial u}{\partial t}\right)^{2}-\frac{1}{2}\left(\frac{\partial u}{\partial x}\right)^{2}-[1-\varepsilon \delta(x)](1-\cos u)+4 h \cos \frac{u}{2}\right\} d x .
$$

The Rayleigh dissipation function has the form

$$
R=\int_{-\infty}^{+\infty} \frac{1}{2} \alpha\left(\frac{\partial u}{\partial t}\right)^{2} d x
$$

The equation of motion for the scalar field $u(x, t)$ taking into account damping in the system takes the following form:

$$
\frac{\partial^{2} u}{\partial t^{2}}-\frac{\partial^{2} u}{\partial x^{2}}+[1-\varepsilon \delta(x)] \sin u+2 h \sin \frac{u}{2}+\alpha \frac{\partial u}{\partial t}=0 .
$$

where $\delta(x)$ is the Dirac delta function, $\varepsilon$ is the constant, $h$ is a value of the external force, $\alpha$ is a parameter of damping. Equation (2.3) is the modified sine-Gordon equation (MSGE).

In the case $\varepsilon=\alpha=h=0$ Eq. (2.3) has a solution in the form of a topological soliton (or kink):

$$
u(x, t)=4 \arctan \left(\exp \left[\Delta\left(v_{0}\right)^{-1}\left(x-v_{0} t\right)\right]\right),
$$

where $\Delta(v)=\left(1-v^{2}\right)^{1 / 2}, v_{0}$ is a continuous parameter $\left(0<v_{0}<1\right)$ determining the velocity of a kink. There is also a solution (2.3) in the form of a spatially localized soliton, namely, a quiescent breather:

$$
u_{\text {breather }}(x, t)=4 \arctan \left(\frac{\sqrt{1-\omega^{2}}}{\omega} \cdot \frac{\sin \omega t}{\cosh \left[\sqrt{1-\omega^{2}}\left(x-x_{0}\right)\right]}\right),
$$

where $\omega$ is the breather frequency and $x_{0}$ is the coordinate of its center.

In the research [5] the case of a "point impurity" was studied in detail $(\varepsilon \delta(x))$ and it was shown that for the corresponding sign of the constant $\varepsilon$ it acts as an attracting potential on a kink, therefore, soliton can be localized. In the case of a spatially extended impurity the kink-impurity interaction was also studied in both undeformable and deformable kink models $[41,46,47]$.

First, we consider the simplest case of point impurity. In this case, for $\varepsilon, h, \alpha \ll 1$, using the method of collective coordinate for the coordinate of the center $X=X(t)$ and the amplitude of localized wave $a=a(t)$ the following system of equations has been obtained (see, e.g., [22]):

$$
\left\{\begin{array}{c}
8 \ddot{X}(t)+U^{\prime}(X)-\varepsilon a(t) F^{\prime}(X)=-8 \dot{X}(t) \alpha+8 h, \\
\ddot{a}(t)+\Omega^{2} a(t)-\frac{\varepsilon^{2}}{2} F(X)=-\dot{a}(t) \alpha,
\end{array}\right.
$$


where

$$
F(X)=2 \frac{\sinh X(t)}{\cosh ^{2} X(t)}, \quad U(X)=8-\frac{2 \varepsilon}{\cosh ^{2} X(t)},
$$

and the frequency of "impurity mode" is determined in the form

$$
\Omega^{2}=1-\frac{\varepsilon^{2}}{4}
$$

The system of equations (2.6) is a system of second-order ordinary differential equations. It is much easier to study than the original equation (2.3). The first equation of (2.6) describes the dynamics of the kink taking into account the presence of the impurity and localized wave on it. The second equation of (2.6) describes the dynamics of a localized wave on an impurity taking into account the interaction of the kink.

\subsection{Nondissipative case}

Consider the problem of the generation of the impurity mode of maximum possible amplitude for the given parameters of the system. It is known that, when a kink is scattered by an impurity, part of its energy is spent on the excitation of a nonlinear wave localized on an impurity or a "breather" (if the kink passes through the impurity) [48, 49]. The value of this energy can vary depending on the initial kink velocity $v_{0}$. Since the frequency of the excited wave is practically independent of $v_{0}$ [50], $v_{0}$ should influence its amplitude. Consider the simplest case, i.e., the case of a point impurity in the framework of the model (2.6) for $h=\alpha=0$. In Fig. 1 the result of the integration is given (2.6) with different modeling parameters, whence it is seen that the breather oscillation amplitude can vary significantly. Suppose that in this model the motion of a kink occurs according to the known law in the form

$$
X_{1}(t)=v_{0} t-10
$$

If we consider the influence of impurities and neglect the only excitation of localized wave by analogy with solution (2.14), the law of motion of a kink can be represented as

$$
X_{2}(t)=\operatorname{arcsinh}\left[\frac{\sqrt{v_{0}^{2}+\varepsilon / 2}}{v_{0}} \cdot \sinh \left(v_{0} t-10\right)\right] .
$$

(a)

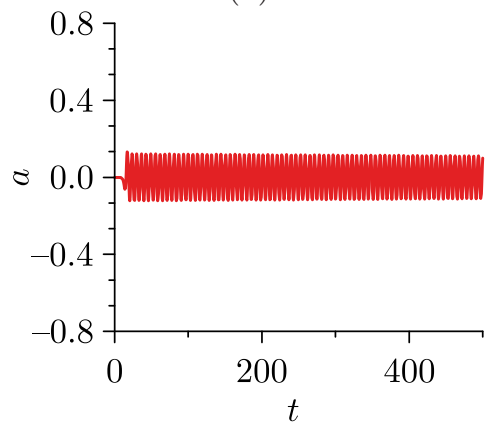

(b)

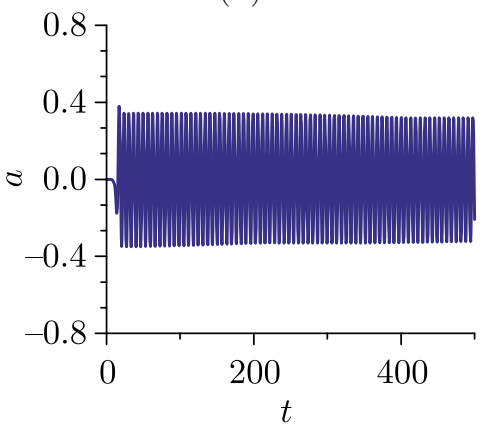

(c)

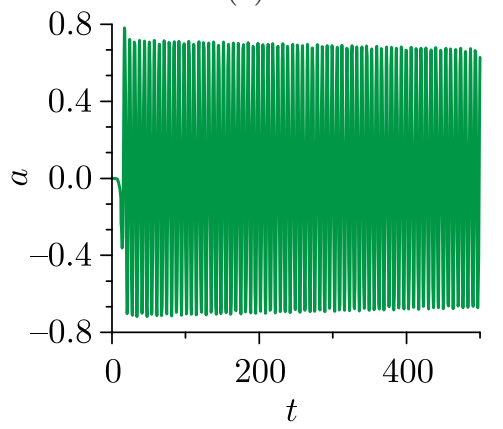

Fig. 1. Time dependence of $a(t)$ calculated by numerical integration (2.6) with initial conditions: $X(0)=-10, \dot{X}(0)=0.7, a(0)=0, \dot{a}(0)=0$ and parameters (a) $\varepsilon=0.3$, (b) $\varepsilon=0.5$, (c) $\varepsilon=0.7$. 
(a)

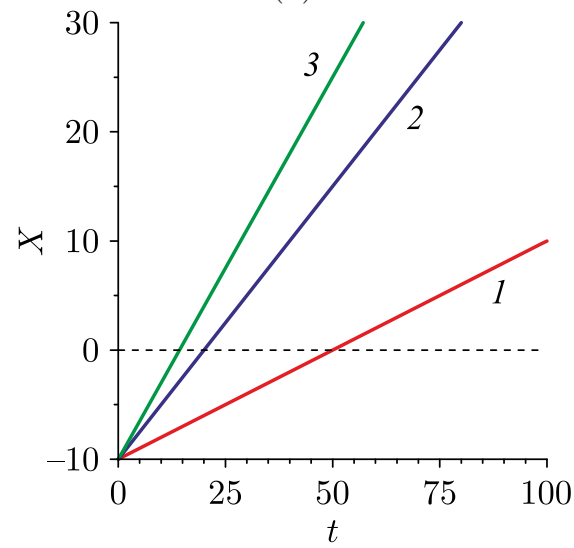

(c)

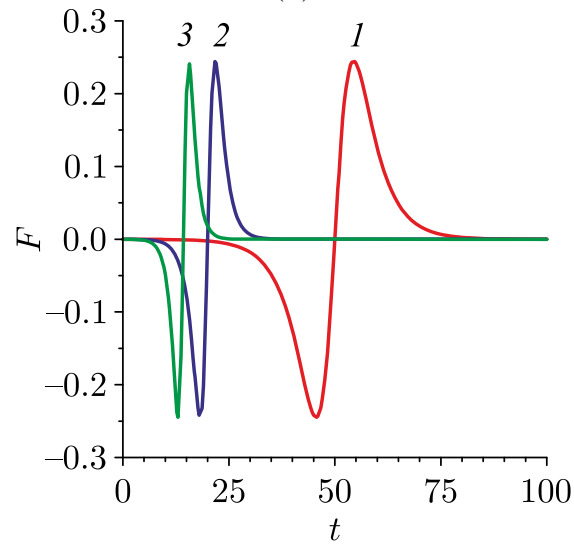

(b)

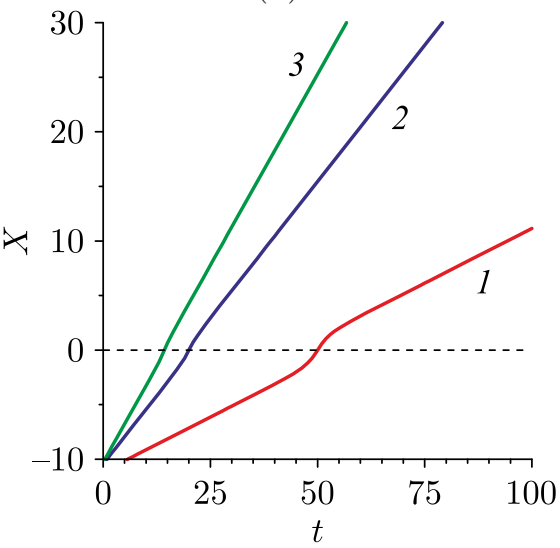

(d)

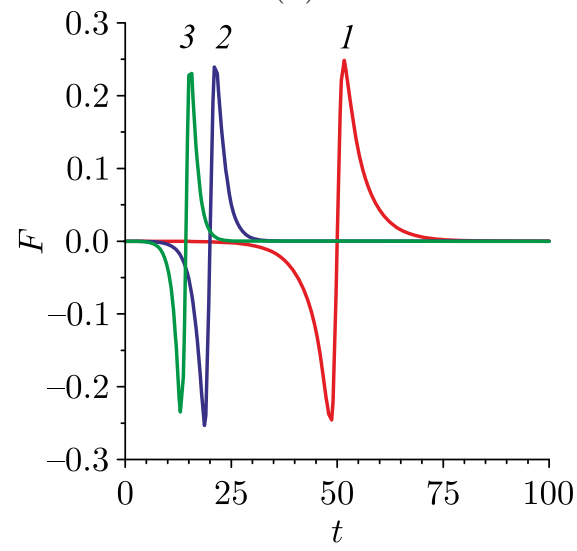

Fig. 2. $X(t)=X_{1}(t)$ (a) and $X(t)=X_{2}(t)$ (b) dependencies from expressions (2.8) and (2.9), respectively, and dependencies $F(t)$ at $X(t)=X_{1}(t)(\mathrm{c})$ and $X(t)=X_{2}(t)$ (d) constructed from the expression (2.11). The parameters are $\varepsilon=0.7$ : 1) $\left.\left.v_{0}=0.2,2\right) v_{0}=0.5,3\right) v_{0}=0.7$.

Figures $2 \mathrm{a}, 2 \mathrm{~b}$ presents the graph of dependence $X(t)$ corresponding to (2.8)-(2.9) which shows that these dependences are slightly different for large values of $v_{0}$ (see curves 3 in Figs. $2 \mathrm{a}, 2 \mathrm{~b}$ ). However, in the region of small values of $v_{0}$ the dependence (2.9) shows a better qualitative correspondence with the results of numerical simulation (see curves 1 in Figs. 2a, 2b).

Since the collective coordinate $X(t)$ is described by the known law, the system (2.6) reduces to one equation for $a(t)$ :

$$
\ddot{a}+\Omega^{2} a=F(t)
$$

where

$$
F(t)=\varepsilon^{2} \frac{\sinh (X(t))}{\cosh ^{2}(X(t))} .
$$

Then this task can be considered as the excitation of a harmonic oscillator by an external force $F(t)$. Figures 2c, 2d present the dependence $F(t)$ for some particular cases. In this case, the initial conditions are assumed as follows:

$$
X(0)=-10, \quad \dot{X}(0)=v_{0}, \quad a(0)=0, \quad \dot{a}(0)=0 .
$$

We use the Runge-Kutta method of numerical integration to study (2.8)-(2.11). The obtained time evolution of $a(t)$ is presented in Fig. 3, which shows that the oscillation amplitude $a(t)$ differs 
(a)

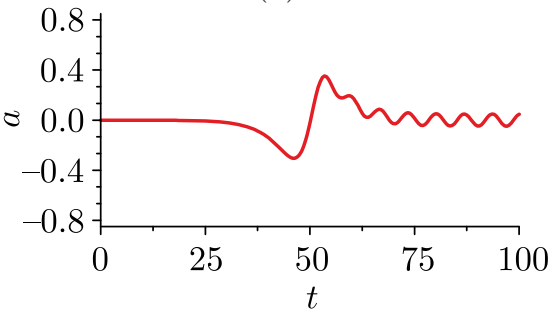

(c)

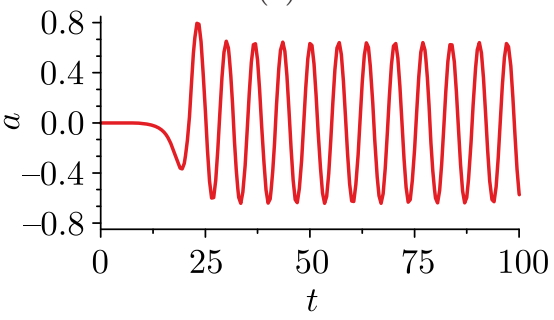

(e)

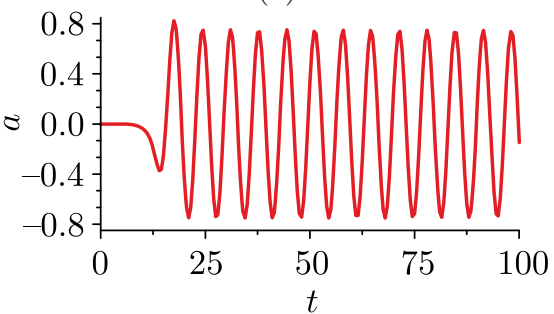

(b)

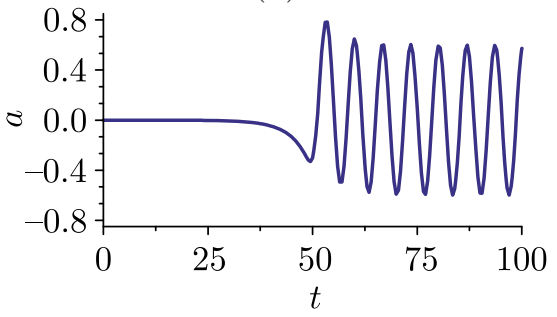

(d)

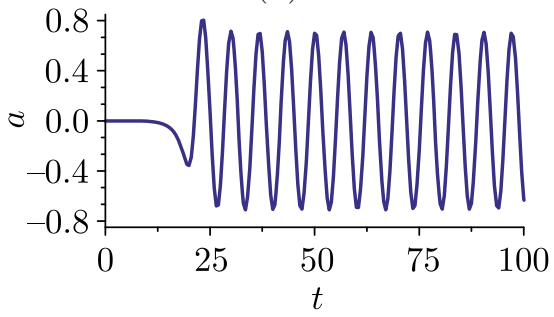

(f)

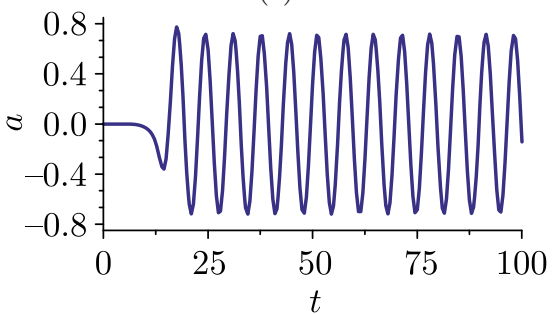

Fig. 3. Time dependence of $a(t)$ calculated by numerical integration (2.10), for the law of motion of a kink (2.8) (a), (c), (e) and (2.9) - (b), (d), (f) and parameters $\varepsilon=0.7$ : (a), (b) $v_{0}=0.2$, (c), (d) $v_{0}=0.5,(\mathrm{e}),(\mathrm{f}) v_{0}=0.7$.

significantly in the case of small $v_{0}$ (Figs. 3a, 3b). We also calculate the maximum breather oscillation amplitude $A_{\max }$ for cases (2.8) and (2.9) and compare it with the values obtained by numerical integration of the original system (2.6). Figure 4 shows that for $v_{0} \rightarrow 1$ when the energy of the excited breather is considerably less than the total energy of a kink, both laws of motion of a kink (2.8) and (2.9) represent well the calculations of the original system (2.6). However, for small $v_{0}$ when the laws of motion differ significantly (see Figs. 2a, 2b, curve 1), the law of motion $X(t)=X_{2}(t)(2.9)$ gives considerably more accurate results. Therefore, it is used in further calculations.

It is possible to find an analytical expression for the breather oscillation amplitude in the case of a point defect using the law of conservation of energy. Then the localized oscillatory mode is given as in [6]:

$$
u(x, t)=B(t) \exp (-\varepsilon|x| / 2),
$$

where $B(t)=a_{0} \cos \left(\Omega t+\theta_{0}\right), \Omega$ is a frequency of the impurity mode $(2.7), \theta_{0}$ being an initial phase. Then the energy stored by the breather can be calculated $[5,51]$ :

$$
E_{i m}=\frac{1}{2} \int_{-\infty}^{+\infty}\left\{\left(\frac{\partial u_{i m}}{\partial t}\right)^{2}+\left(\frac{\partial u_{i m}}{\partial x}\right)^{2}+[1-\varepsilon \delta(x)] u_{i m}^{2}\right\} d x=\frac{\Omega^{2} a_{0}^{2}}{\varepsilon}
$$


(a)

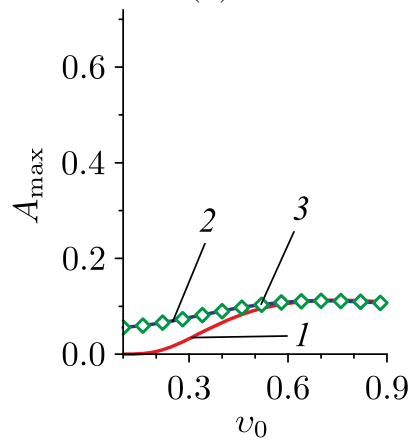

(b)

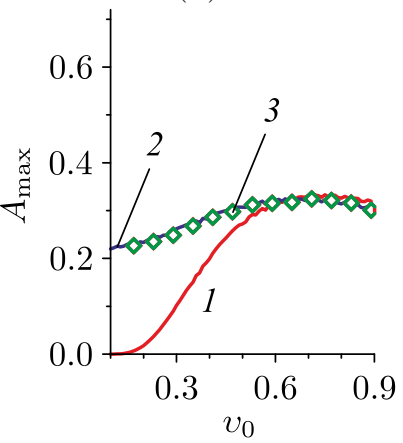

(c)

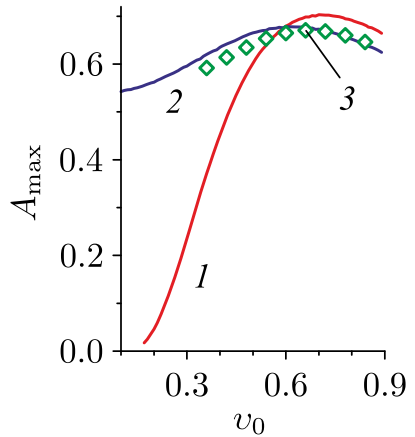

Fig. 4. Maximum breather oscillation amplitude $A_{\max }$ depending on the initial kink velocity $v_{0}$ at $\varepsilon=0.3(\mathrm{a}), \varepsilon=0.5$ (b) and $\varepsilon=0.7$ (c), calculated by numerical integration: 1) Eqs. (2.10) for the law of motion of a kink (2.8), 2) Eqs. (2.10) for the law of motion of a kink (2.9), 3) systems of equations (2.6).

Suppose that the variation $X(t)$ is described by the solution [6]:

$$
X(t)=\operatorname{arcsinh}\left(\gamma \sinh \left(v_{0} t\right)\right)
$$

where $\gamma=\sqrt{1+\varepsilon /\left(2 v_{0}^{2}\right)}$. Then the force acting on the breather (oscillator) is given by the expression (taking into account (2.11)):

$$
f(t)=\frac{\varepsilon^{2} A \sinh \left(v_{0} t\right)}{1+A^{2} \sinh \left(v_{0} t\right)} .
$$

If the complex variable $\xi(t)=\dot{a}+i \Omega t$ is used, then the equation of oscillations (2.10) can be reduced to the form

$$
\dot{\xi}-i \Omega \xi(t)=f(t)
$$

which has the solution [51]:

$$
\xi(t)=e^{i \Omega t} \int_{-\infty}^{t} f(\tau) e^{-i \Omega \tau} d \tau,
$$

with initial conditions $a(-\infty)=\dot{a}(-\infty)=0$, i.e., the oscillator (breather) is absent before the interaction. The total energy transferred from the particle (kink) to the oscillator (breather) can be found as in [51]:

$$
E_{i m}=2 \pi^{2} \varepsilon^{2} \sinh ^{2}\left[\frac{\Omega}{2 v_{0}} \arccos \left(\frac{2 v_{0}^{2}-\varepsilon}{2 v_{0}^{2}+\varepsilon}\right)\right] \cosh ^{-2}\left(\frac{\Omega \pi}{2 v_{0}}\right) .
$$

Then we obtain the following expression from (2.13) and (2.18) for the breather amplitude:

$$
a_{0}^{2}=\frac{2 \pi^{2} \varepsilon^{3}}{\Omega^{2}} \sinh ^{2}\left[\frac{\Omega}{2 v_{0}} \arccos \left(\frac{2 v_{0}^{2}-\varepsilon}{2 v_{0}^{2}+\varepsilon}\right)\right] \cosh ^{-2}\left(\frac{\Omega \pi}{2 v_{0}}\right) .
$$

The expression (2.19) describes well the results obtained by numerical integration of the system (2.6) (Fig. 5). However, the dependences obtained are very far from the breather amplitude of the model (2.3). It was calculated by the numerical method described in [21]. Nevertheless, 
(a)

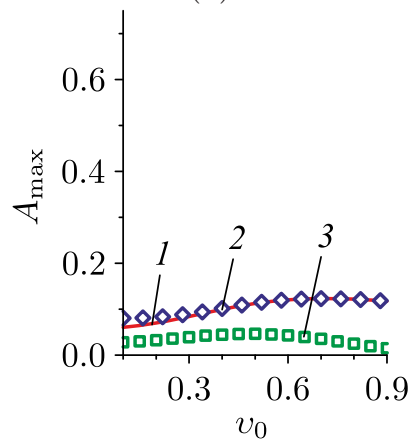

(b)

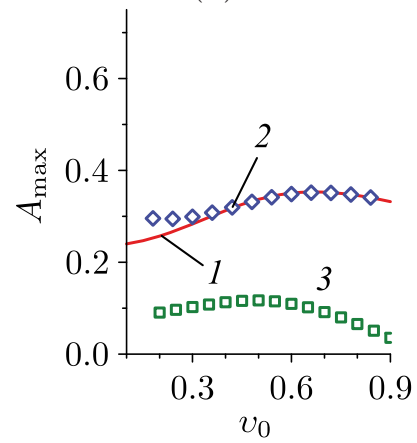

(c)

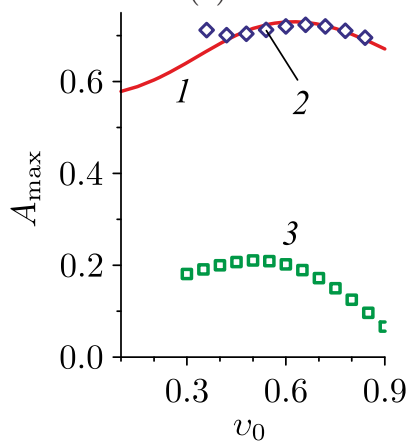

Fig. 5. Maximum breather oscillation amplitude $A_{\max }$ depending on the initial kink velocity $v_{0}$ at $\varepsilon=$ $=0.3$ (a), $\varepsilon=0.5$ (b) and $\varepsilon=0.7$ (c), calculated: 1) by analytical expressions (2.19), 2) by numerical integration of the system of equations (2.6), 3) numerical simulation of Eq. (2.3).

(a)

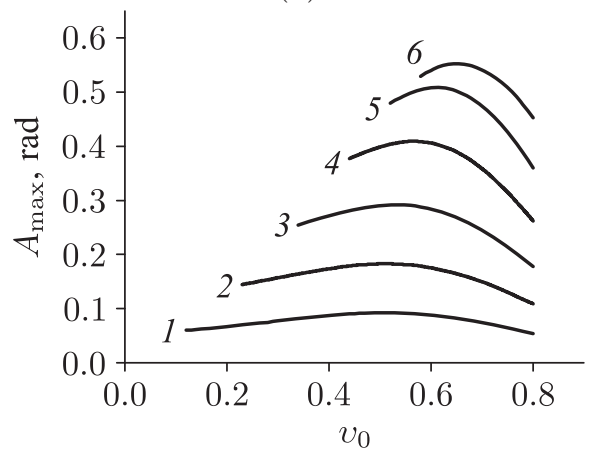

(b)

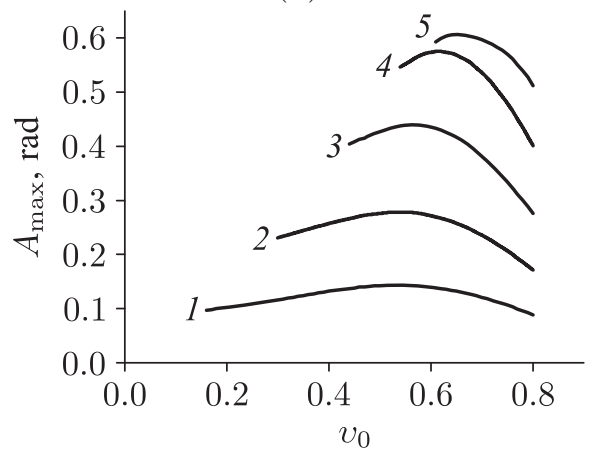

Fig. 6. Maximum breather amplitude $A_{\max }$ in the center of the extended impurity depending on the initial kink velocity $v_{0}$ at $W=1$ (a) and $W=1.5$ (b) in the framework of the model (2.20). Curves 1-6 correspond to the cases $\Delta K=0.5,0.75,1,1.25,1.5,1.75$, respectively.

the nature of the dependences is qualitatively the same: one maximum is present in all the curves in Fig. 5, which is presumably related to the ratio of the shape of the external force $F(t)$ and the shape of the potential $U(X)$. Moreover, the maximum point depends on the parameters of the impurity. Similar behavior is observed for the case of extended impurities of rectangular form with a width $W$ and a depth $\Delta K,(\Delta K=0$ at $x<0, x>W)$ :

$$
\frac{\partial^{2} u}{\partial t^{2}}-\frac{\partial^{2} u}{\partial x^{2}}+[1-\Delta K] \sin u+2 h \sin \frac{u}{2}+\alpha \frac{\partial u}{\partial t}=0 .
$$

Figure 6 shows the maximum amplitude of the generated breather obtained numerically from Eq. (2.20). It is also shown that all curves have one maximum. The change in amplitude in this case is more significant than in the case of a point impurity.

\subsection{Interaction of a kink with an impurity mode taking into account dissipation and external force}

Consider the solution of the system of equations (2.6) in the presence of damping and external force. Since parameter $h$ is absent in the second equation of the system (2.6) (due to the chosen approximation for the effective Lagrangian), the action of the external force does 
(a)

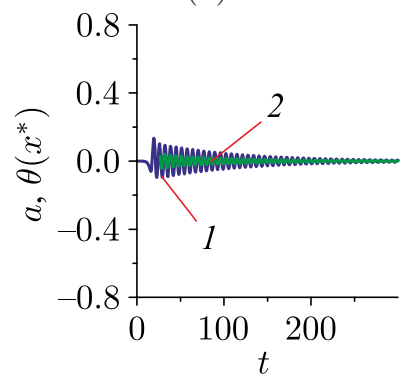

(b)

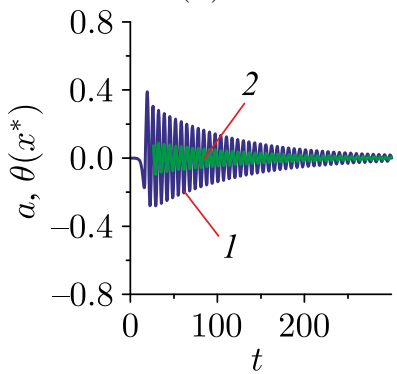

(c)

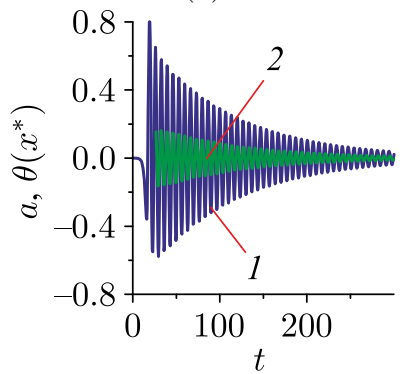

Fig. 7. Time evolution of the collective coordinate $a(t)$ (curve 1) calculated from (2.6) and $\theta\left(x^{*}\right)$ (curve 2) calculated from (2.3), for the case where the kink crosses by inertia the impurity with center at the point $x^{*}=0$. The simulation parameters are $v_{0}=0.7, \alpha=0.02$ : (a) $\varepsilon=0.3$, (b) $\varepsilon=0.5$, (c) $\varepsilon=0.7$.

not affect the oscillations of the impurity mode within this analytical model. Therefore, in this section, we restrict ourselves to the assumption that the kink moving by inertia passes through the impurity region.

Figure 7 presents the result of the integration of the analytical model (2.6) (curves 1) and the initial equation (2.3) (curves 2) for three different values of $\varepsilon$. It shows that the oscillation amplitude decreases under the effect of damping and the curves 1 and 2 are qualitatively the same. However, a quantitative comparison of their amplitude varies several times with increasing parameter $\varepsilon$.

Analysis of the analytical model (in the absence of dissipation) has shown that the excitation of an impurity mode as a result of the scattering of a kink on an impurity can be considered as the excitation of an effective oscillator by a short-term impulse $F(t)$. Figures $2 \mathrm{c}, 2 \mathrm{~d}$ present an example of an approximate view of the dependence $F(t)$, where the graphs of this dependence are given for particular cases of the laws of motion of a kink $X(t)$. Thus, after the impurity mode is excited $F(t) \rightarrow 0$ further its oscillations occur under the influence of damping in the absence of external influence. Then the second equation of the system (2.6) can be represented (in our case) in the form

$$
\ddot{a}(t)+\Omega^{2} a(t)=-\dot{a}(t) \alpha,
$$

which is a well-known equation of oscillations of a harmonic oscillator with damping [52], its solution can be represented in the form

$$
a(t)=B(t) \cos \left(\Omega t+\phi_{0}\right), \quad B(t)=B_{0} \exp \left(-\frac{\alpha t}{2}\right)
$$

where $\phi_{0}$ is an initial phase and $B(t)$ is an amplitude of the oscillations. Then the logarithmic decrement of damping $\lambda$ of the dependence $a(t)$ taking into account the expression $\Omega^{2}=1-\frac{\varepsilon^{2}}{4}$ can be found as follows:

$$
\lambda=\frac{\alpha \pi}{\Omega}=\frac{\alpha \pi}{\sqrt{1-\varepsilon^{2} / 4}} .
$$

The formulas (2.22)-(2.23) describe sufficiently accurately the dependence $a(t)$ only from a particular moment of time. Figure 8 shows that the oscillation amplitude of the dependence $a(t)$ (solid curves) coincides quantitatively with the analytical expression for $B(t)$ (dashed curves), starting from the third oscillation period, since the effect of a nonzero value of the 
(a)

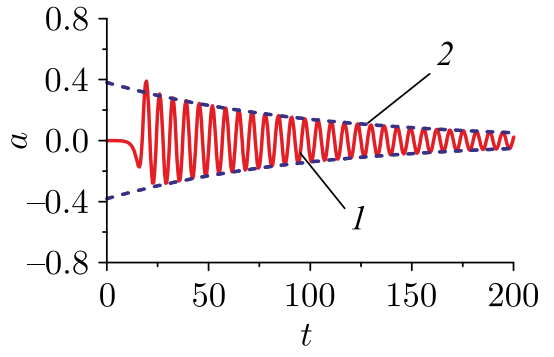

(b)

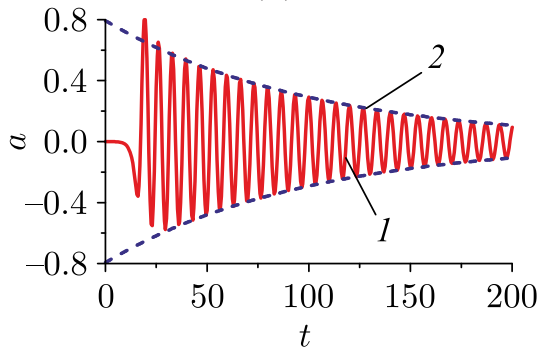

Fig. 8. Curve 1 is a time evolution of collective coordinate of $a(t)$ from (2.6) for the case where the kink crosses by inertia the impurity at $v_{0}=0.7, \alpha=0.02$ : a) $\varepsilon=0.5$, b) $\varepsilon=0.7$. Curve 2 is a dependence of $B(t)$ calculated by the formula (2.22) at $\alpha=0.02$ : (a) $B_{0}=0.38169$, (b) $B_{0}=0.79306$.

impulse $F(t)$ is still manifested in the first two periods. Therefore, the initial part of the dependence should be excluded from the analysis when calculating the decrement of damping directly (for example, for the model (2.3)).

Next calculate the logarithmic decrement of damping $\lambda$ of the oscillations of the impurity mode within the framework of the initial equation (2.3). Figure 9 presents the dependence $\lambda(\varepsilon)$, calculated by integration of Eq. (2.3) (solid curve 1) and the analytical dependence, constructed using the formula (2.23) (dashed curve 2). This figure shows that the curves obtained have a good quantitative agreement. The error of the curve is due to the fact that the amplitude of the excited impurity mode is extremely low at small values of $\varepsilon$ (see Fig. 7). Therefore, the relative error in calculating the amplitude of the impurity mode is significant.

Thus, the effect of damping on the impurity mode is almost identical to the model (2.3) despite admitted approximation used in the construction of analytical models of (2.6). This suggests that the dissipation of the system, which takes into account the waste of energy on the excitation of the impurity mode, is one of the main damping channels (at least for the point impurities under consideration). However, it is possible that the radiation emitted by a localized wave can also play a significant role for large oscillation amplitudes of the impurity modes. Similar assumptions are discussed, for example, in [50, 53].

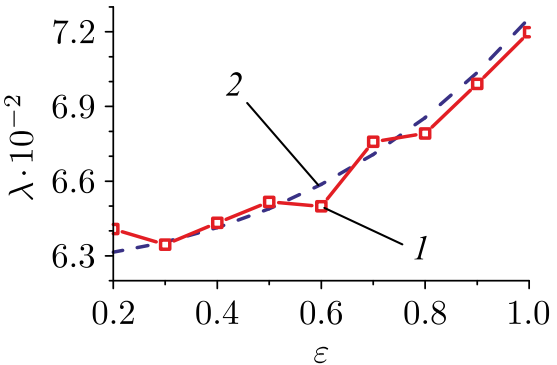

Fig. 9. Logarithmic decrement of damping $\lambda$ depending on $\varepsilon$ when $\alpha=0.02$, $v_{0}=0.7$. Curve 1 is calculated in the integration of Eq. (2.3). Curve 2 is built according to the formula (2.23).

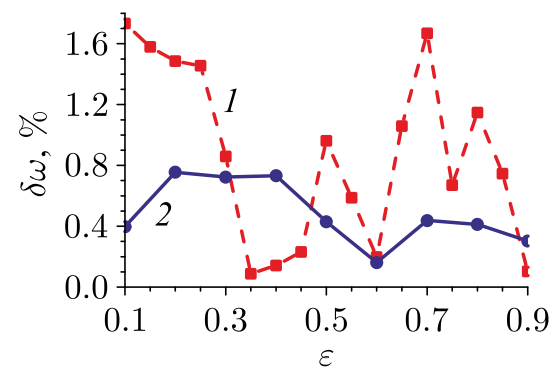

Fig. 10. Dependence of the relative deviation of the oscillation frequency of the impurity mode $\delta \omega$ for a point impurity on the parameter $\varepsilon$ at $\alpha=0.02$. The deviation is calculated from the exact value, which is determined by the formula (2.7). The impurity mode is excited as the kink moves by inertia (curve 1) under the action of an external force (curve 2) $h=0.0196039$ $\left(v_{0}=0.7\right)$. 
The significant difference in the amplitude of the excited impurity modes in both models (Fig. 7) is due to the approximations allowed in the derivation of (2.3).

The effect of an external force can have a certain effect on the oscillations of the impurity mode as it was shown in [54]. For example, the action of the field can lead to a change in the oscillation frequency, however, this change may be insignificant in the case of a small external force. Two graphs of the dependence of the relative error in the oscillation frequency of the impurity mode for the cases of motion of the kink under the action of an external force (curve 1) and by inertia (curve 2) are shown for comparison in Fig. 10. The occurrence of damping did not affect considerably the deviation of the frequency of the impurity mode $\delta \omega$ from the nondissipative case (in both cases $\delta \omega<1 \%$ ). Also, Fig. 10 shows that the relative deviation of the frequency of the impurity mode from the nondissipative case does not exceed $2 \%$ for the case of motion of the kink under the action of an external force $h=0.0196039$. Thus, the presence of a small external force has no significant effect on the oscillations of the impurity mode. The approximation in the analytical model, when $h$ is absent in the second equation of (2.6), is justified.

\section{Conclusion}

It was shown that the scattering of a kink on an impurity can be considered as an effective way to excite localized nonlinear waves. The dependence of the breather amplitude on the initial kink velocity when there is only one maximum was calculated for both point and extended impurities. This allows controlling the excitation energy of localized waves using the initial kink velocity and impurity parameters. This behavior remains qualitatively the same in the presence of a small external force and damping. The analytical expression for the decrement of damping was calculated. It is shown that the considered small values of the external force do not have a significant effect on the oscillations of localized waves. This opens up the possibility of using this method of generation of localized impurities in real physical systems. A small external force can be used to set the initial kink velocity.

\section{References}

[1] The Sine-Gordon Model and Its Applications: From Pendula and Josephson Junctions to Gravity and High-Energy Physics, J. Cuevas-Maraver, P. Kevrekidis, F. Williams (Eds.), Cham: Springer, 2014.

[2] Encyclopedia of Nonlinear Science, A. Scott (Ed.), New York: Routledge, 2005.

[3] Yakushevich, L.V., Savin, A.V., and Manevitch, L.I., On the Internal Dynamics of Topological Solitons in DNA, Phys. Rev. E, 2002, vol.66, no. 1, 016614, 14 pp.

[4] Shamsutdinov, M. A., Khabibullin, I. T., Kharisov, A. T., and Tankeyev, A.P., Dynamics of Magnetic Kinks in Exchange-Coupled Ferromagnetic Layers, Phys. Metals Metallogr., 2009, vol.108, no. 4, Art. 327; see also: Fiz. Met. i Metalloved., 2009, vol. 108, no.4, pp. 345-358.

[5] Braun, O.M. and Kivshar, Yu.S., The Frenkel-Kontorova Model: Concepts, Methods, and Applications, Berlin: Springer, 2004.

[6] Dauxois, Th. and Peyrard, M., Physics of Solitons, Cambridge: Cambridge Univ. Press, 2010.

[7] Gani, V.A., Lensky, V., and Lizunova, M. A., Kink Excitation Spectra in the (1+1)-Dimensional $\varphi^{8}$ Model, J. High Energ. Phys., 2015, vol. 2015, no. 8, Art. 147, 21 pp.

[8] Gani, V.A., Lizunova, M. A., and Radomskiy, R.V., Scalar Triplet on a Domain Wall, J. Phys. Conf. Ser., 2016, vol. 675, 012020, 4 pp. 
[9] Gani, V. A., Kudryavtsev, A. E., and Lizunova, M. A., Kink Interactions in the (1+1)-Dimensional $\varphi^{6}$ Model, Phys. Rev. D, 2014, vol. 89, no. 12, 125009, 12 pp.

[10] Kavitha, L., Parasuraman, E., Gopi, D., Prabhu, A., and Vicencio, R. A., Nonlinear Nano-Scale Localized Breather Modes in a Discrete Weak Ferromagnetic Spin Lattice, J. Magn. Magn. Mater., 2016, vol. 401, pp. 394-405.

[11] Kavitha, L., Mohamadou, A., Parasuraman, E., Gopi, D., Akila, N., and Prabhu, A., Modulational Instability and Nano-Scale Energy Localization in Ferromagnetic Spin Chain with Higher Order Dispersive Interactions, J. Magn. Magn. Mater., 2016, vol. 404, pp. 91-118.

[12] Golovchan, A. V., Kruglyak, V.V., Tkachenko, V.S., and Kuchko, A. N., Magnonic Band Spectrum of Spin Waves in an Elliptical Helix, R. Soc. Open Sci., 2018, vol. 5, no. 1, 172285, 9 pp.

[13] Askari, A., Saadatmand, D., and Javidan, K., Collective Coordinate System in $(2+1)$ Dimensions: $C P^{1}$ Lumps-Potential Interaction, Waves Random Complex Media, 2019, vol. 29, no. 2, pp. 368-381.

[14] Moradi Marjaneh, A., Askari, A., Saadatmand, D., and Dmitriev, S. V., Extreme Values of Elastic Strain and Energy in Sine-Gordon Multi-Kink Collisions, Eur. Phys. J. B, 2018, vol.91, no. 1, Paper No. 22, 8 pp.

[15] Popov, S. P., Compactons and Riemann Waves of an Extended Modified Korteweg-de Vries Equation with Nonlinear Dispersion, Comput. Math. Math. Phys., 2018, vol. 58, no. 3, pp. 437-448.

[16] Popov, S.P., Nonautonomous Soliton Solutions of the Modified Korteweg-de Vries-Sine-Gordon Equation, Comput. Math. Math. Phys., 2016, vol. 56, no.11, pp. 1929-1937.

[17] Dubovik, M. N., Korzunin, L. G., and Filippov, B. N., Asymmetrical Pinning of Vortex Domain Walls in Ferromagnetic Films in Areas with Increased Saturation Magnetization, Phys. Metals Metallogr., 2015, vol. 116, no. 7, pp. 656-662; see also: Fiz. Met. i Metalloved., 2015, vol. 116, no. 7, pp. 694-700.

[18] Ekomasov, E. G., Gumerov, A. M., Kudryavtsev, R. V., Dmitriev, S. V., and Nazarov, V. N., Multisoliton Dynamics in the Sine-Gordon Model with Two Point Impurities, Braz. J. Phys., 2018, vol. 48, no. 6, pp. 576-584.

[19] Nonlinear Science at the Dawn of the 21st Century, P. L. Christiansen, M. P. Sørensen, A. C. Scott (Eds.), Lecture Notes in Phys., vol. 542, Berlin: Springer, 2000.

[20] Currie, J. P., Trullinger, S. E., Bishop, A. R., and Krumhandl, J. A., Numerical Simulation of SineGordon Soliton Dynamics in the Presence of Perturbations, Phys. Rev. B, 1977, vol.15, no. 12, pp. $5567-5580$.

[21] Ekomasov, E. G., Gumerov, A.M., and Murtazin, R. R., Interaction of Sine-Gordon Solitons in the Model with Attracting Impurities, Math. Models Methods Appl. Sci., 2016, vol.40, no. 17, pp. 6178-6186.

[22] Ekomasov, E. G., Gumerov, A. M., and Kudryavtsev, R. V., On the Possibility of the Observation of the Resonance Interaction between Kinks of the Sine-Gordon Equation and Localized Waves in Real Physical Systems, JETP Lett., 2015, vol.101, no.12, pp. 835-839; see also: Pis'ma v Zh. Èksper. Teoret. Fiz., 2015, vol. 101, no. 12, pp. 935-939.

[23] Ekomasov, E. G., Gumerov, A.M., and Kudryavtsev, R.V., Resonance Dynamics of Kinks in the Sine-Gordon Model with Impurity, External Force and Damping, J. Comput. Appl. Math., 2017, vol. 312, pp. 198-208.

[24] Kivshar, Yu. S., Pelinovsky, D. E., Cretegny, T., and Peyrard, M., Internal Modes of Solitary Waves, Phys. Rev. Lett., 1998, vol. 80, no. 23, pp. 5032-5035.

[25] Kivshar, Yu. S., Malomed, B.A., Zhang, F., and Vazquez, L., Creation of Sine-Gordon Solitons by a Pulse Force, Phys. Rev. B, 1991, vol. 43, no. 1, pp. 1098-1109.

[26] González, J. A., Bellorín, A., and Guerrero, L. E., Internal Modes of Sine-Gordon Solitons in the Presence of Spatiotemporal Perturbations, Phys. Rev. E (3), 2002, vol.65, no. 6, 065601, 4 pp.

[27] Kivshar, Yu. S. and Malomed, B. A., Addendum: Dynamics of Solitons in Nearly Integrable Systems, Rev. Mod. Phys., 1991, vol.63, no. 1, pp. 211-212.

[28] Javidan, K., Analytical Formulation for Soliton-Potential Dynamics, Phys. Rev. E, 2008, vol.78, no. 4, 046607, 8 pp. 
[29] Chacón, R., Bellorín, A., Guerrero, L. E., and González, J. A., Spatiotemporal Chaos in Sine-Gordon Systems Subjected to Wave Fields: Onset and Suppression, Phys. Rev. E, 2008, vol. 77, no. 4, 046212, 4 pp.

[30] González, J. A., Bellorín, A., Reyes, L. I., Vásquez, C., and Guerrero, L. E., Geometrical Resonance in Spatiotemporal Systems, Europhys. Lett., 2003, vol. 64, no. 6, pp. 743-749.

[31] González, J.A., Cuenda, S., and Sánchez, A., Kink Dynamics in Spatially Inhomogeneous Media: The Role of Internal Modes, Phys. Rev. E (3), 2007, vol. 75, no. 3, 036611, 7 pp.

[32] Gumerov, A.M., Ekomasov, E. G., Murtazin, R.R., and Nazarov, V.N., Transformation of sineGordon solitons in models with variable coefficients and damping, Comput. Math. Math. Phys., 2015, vol. 55, no. 4, pp. 628-637.

[33] González, J.A., Bellorín, A., García-Ñustes, M. A., Guerrero, L. E., Jiménez, S., and Vázquez, L., Arbitrarily Large Numbers of Kink Internal Modes in Inhomogeneous Sine-Gordon Equations, Phys. Lett. A, 2017, vol. 381, no. 24, pp. 1995-1998.

[34] González, J. A. Jiménez, S., Bellorín, A., Guerrero, L. E., and Vázquez, L., Internal Degrees of Freedom, Long-Range Interactions and Nonlocal Effects in Perturbed Klein-Gordon Equations, Phys. A, 2012, vol. 391, no. 3, pp. 515-527.

[35] Saadatmand, D., Dmitriev, S. V., Borisov, D. I., and Kevrekidis, P. G., Interaction of Sine-Gordon Kinks and Breathers with a Parity-Time-Symmetric Defect, Phys. Rev. E, 2014, vol.90, no.5, 052902, 10 pp.

[36] Belova, T.I. and Kudryavtsev, A.E., Solitons and Their Interactions in Classical Field Theory, Physics-Uspekhi, 1997, vol.40, no.4, pp.359-386; see also: Uspekhi Fiz. Nauk, 1997, vol.167, no. 4, pp. 377-406.

[37] Popov, S. P., Influence of Dislocations on Kink Solutions of the Double Sine-Gordon Equation, Comput. Math. Math. Phys., 2013, vol. 53, no. 12, pp. 1891-1899.

[38] Malomed, B. A., Dynamics of Quasi-One-Dimensional Kinks in the Two-Dimensional Sine-Gordon Model, Phys. D, 1991, vol. 52, nos. 2-3, pp. 157-170.

[39] Saadatmand, D. and Javidan, K., Collective-Coordinate Analysis of Inhomogeneous Nonlinear Klein-Gordon Field Theory, Braz. J. Phys., 2013, vol.43, nos. 1-2, pp. 48-56.

[40] Ekomasov, E. G., Gumerov, A.M., Murtazin, R. R., Kudryavtsev, R. V., Ekomasov, A.E., and Abakumova, N. N., Resonant Dynamics of the Domain Walls in Multilayer Ferromagnetic Structure, Solid State Phenom., 2015, vol. 233-234, pp. 51-54.

[41] Ekomasov, E. G. and Shabalin, M. A., Simulation the Nonlinear Dynamics of Domain Walls in Weak Ferromagnets, Phys. Metals Metallogr., 2006, vol. 101, Suppl. 1, pp. S48-S50.

[42] Ekomasov, E. G., Murtazin, R. R., Bogomazova, O. B., and Gumerov, A. M., One-Dimensional Dynamics of Domain Walls in Two-Layer Ferromagnet Structure with Different Parameters of Magnetic Anisotropy and Exchange, J. Magn. Magn. Mater., 2013, vol. 339, pp. 133-137.

[43] Ekomasov, E. G., Murtazin, R. R., Bogomazova, O. B., and Nazarov, V. N., Excitation and Dynamics of Domain Walls in Three-Layer Ferromagnetic Structure with Different Parameters of the Magnetic Anisotropy and Exchange, Mater. Sci. Forum, 2016, vol. 845, pp. 195-198.

[44] Gulevich, D. R. and Kusmartsev, F. V., Perturbation Theory for Localized Solutions of the SineGordon Equation: Decay of a Breather and Pinning by a Microresistor, Phys. Rev. B, 2006, vol. 74, no. 21, 214303, 5 pp.

[45] Gumerov, A. M., Ekomasov, E. G., Kudryavtsev, R. V., and Fakhretdinov, M. I., Localized Magnetic Inhomogeneities Generation on Defects As a New Channel of Damping for a Moving Domain Wall, Letters on Materials, 2018, vol.8, no.3, pp. 299-304 (Russian).

[46] Paul, D. I., Soliton Theory and the Dynamics of a Ferromagnetic Domain Wall, J. Phys. C, 1979, vol. 12 , no. 3, pp. 585-593.

[47] Piette, B. and Zakrzewski, W. J., Scattering of Sine-Gordon Kinks on Potential Wells, J. Phys. A, 2007, vol. 40, no. 22, pp. 5995-6010.

[48] Gumerov, A. M. and Ekomasov, E. G., Study of the Effect of Point Defects on the Nonlinear Dynamics of Magnetic Nonuniformities, Letters on Materials, 2013, vol. 3, no. 2, pp. 103-105 (Russian). 
[49] Ekomasov, E. G., Gumerov, A.M., and Kudryavtsev, R. V., Dynamics of Localized Magnetic Inhomogeneities in the Five-Layer Ferromagnetic Structure, Letters on Materials, 2016, vol.6, no. 2, pp. 138-140 (Russian).

[50] Ekomasov, E. G., Azamatov, S. A., and Murtazin, R. R., Studying the Nucleation and Evolution of Magnetic Inhomogeneities of the Soliton and Breather Type in Magnetic Materials with Local Inhomogeneities of Anisotropy, Phys. Metals Metallogr., 2008, vol. 105, no.4, pp. 313-321; see also: Fiz. Met. i Metalloved., 2008, vol. 105, no. 4, pp. 341-349.

[51] Zhang, F., Kivshar, Yu. S., and Vazquez, L., Resonant Kink-Impurity Interactions in the SineGordon Model, Phys. Rev. A, 1992, vol.45, no. 8, pp.6019-6030.

[52] Landa, P.S., Nonlinear Oscillations and Waves in Dynamical Systems, Math. Appl., vol.360, Dordrecht: Springer, 2013.

[53] Goodman, R. H., Holmes, P. J., and Weinstein, M. I., Interaction of Sine-Gordon Kinks with Defects: Phase Space Transport in a Two-Mode Model, Phys. D, 2002, vol. 161, no. 1, pp. 21-44.

[54] Ekomasov, E. G., Murtazin, R. R., and Nazarov, V. N., Excitation of Magnetic Inhomogeneities in Three-Layer Ferromagnetic Structure with Different Parameters of the Magnetic Anisotropy and Exchange, J. Magn. Magn. Mater., 2015, vol. 385, pp. 217-221. 